\title{
Spontaneous Healing of Acromial Stress Fracture Caused by Clavicle Hook Plate in Acromioclavicular Joint Dislocation - A Case Report
}

\author{
Gang-Un Kim, Seong-Hwan Kim, Jae-Sung Lee, Jae Yoon Kim \\ Department of Orthopaedic Surgery, Chung-Ang University College of Medicine, Seoul, Korea
}

\begin{abstract}
Clavicular hook plate is known to be an effective treatment on acromioclavicular (AC) joint injury, but there have been some reports of complications, like osteolysis and bony erosion of the undersurface of acromion. Fifty-five year old male underwent open reduction and hook plate insertion on Rockwood type 5 acromioclavicular joint dislocation. He complained of protrusion of posterior acromion at 1 month after the surgery, and acromial fracture was noted in simple radiographs. The hook plate was removed and any other treatment for osteosynthesis was refused by the patient. At the 18 months after the surgery, the patient had no pain and a full range of motion with no tenderness around the shoulder joint. After two years, plain radiographs revealed complete bony union of the acromion fracture.
\end{abstract}

(Clin Shoulder Elb 2014;17(1):36-39)

Key Words: Acromioclavicular dislocation; Hook plate; Acromial fracture

Acromioclavicular (AC) joint injuries are commonly seen after trauma affecting the shoulder. They are usually classified as Rockwood type III, IV and V dislocations and nearly always require operative treatment. ${ }^{1)}$ There are a variety of surgical approaches to treat these injuries, and there is controversy regarding which is the most appropriate method to stabilize the AC joint. Among the various approaches, clavicular hook plate fixation for AC dislocation is increasing in popularity. The clavicular hook plate is an implant that permits retention in the transverse plane, stabilizes the joint, and allows for joint movement early in the healing process. ${ }^{2)}$

There are some studies that report post-operative complications after clavicular hook plate implantation, such as osteolysis, subacromial impingement and bony erosion. ${ }^{2,3)}$ Here, we report the spontaneous healing of a displaced acromial fracture after removal of an implanted clavicular hook plate.

\section{Case Report}

A 55-year-old male presented at our outpatient clinic with a painful right shoulder after a fall on his right side from a height of two meters. A plain radiograph after the initial injury did not indicate that there was a fracture nor did it show any evidence of an acromioclavicular joint injury. One month after the trauma, the patient visited the clinic again with a painful deformity in the right shoulder that appeared a few days prior. Plain radiograph revealed Rockwood type $\mathrm{V}$ acromioclavicular joint dislocation (Fig. 1). He underwent open reduction and internal fixation of the dislocated AC joint with a $3.5 \mathrm{~mm}$ LCP Clavicle Hook Plate (6 holes with $15 \mathrm{~mm}$ depth; Synthes, West Chester, PA, USA). The torn coracoclavicular ligaments were left without any repair or reconstruction. After operation, plain radiograph showed proper reduction and firm fixation of the AC joint (Fig. 2).

The post-operative treatment plan called for the shoulder to be held in an immobilizer for six weeks, and passive rangeof-motion exercises started three weeks after surgery. After one week of passive shoulder exercise, the patient complained of

Received March 15, 2014. Revised March 19, 2014. Accepted March 19, 2014.

Correspondence to: Jae Yoon Kim

Department of Orthopedic Surgery, Chung-Ang University College of Medicine, 102 Heukseok-ro, Dongjak-ku, Seoul 156-755, Korea

Tel: +82-2-6299-1592, Fax: +82-2-822-1710, E-mail: kjycje@gmail.com

Financial support: None. Conflict of interests: None. 


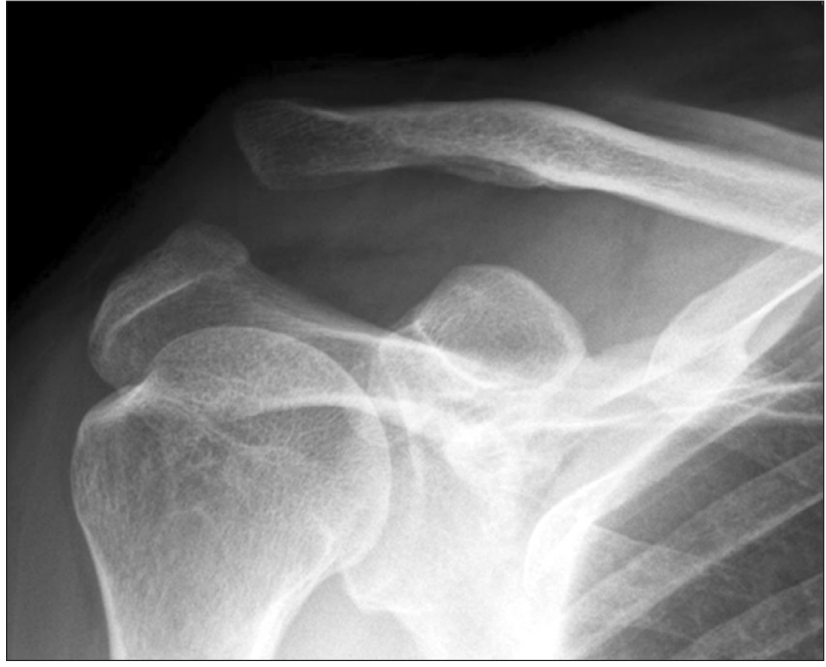

Fig. 1. Rockwood type V acromioclavicular dislocation was found on plain radiograph.

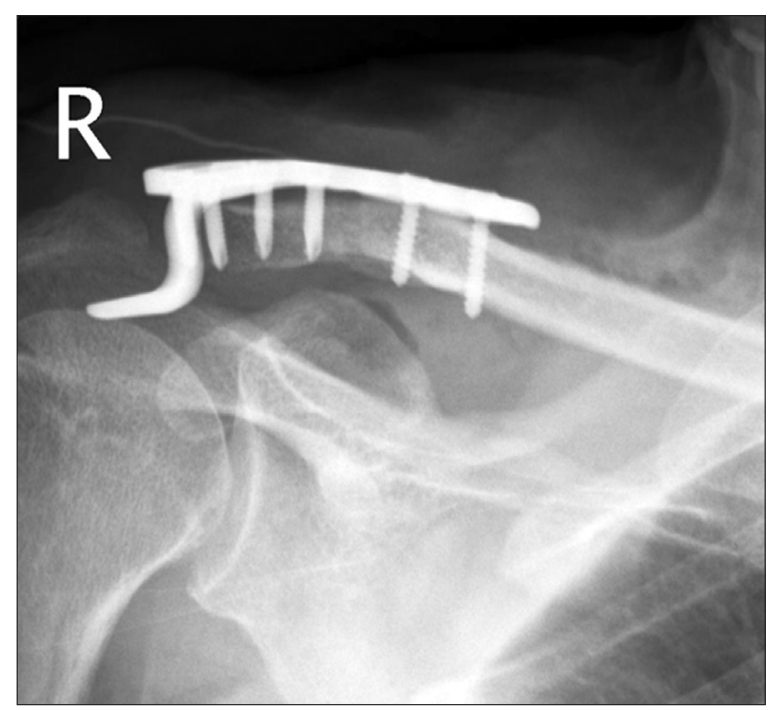

Fig. 2. Immediate postoperative radiograph showed a well-reduced acromioclavicular joint.

pain in the right shoulder; he reported no further trauma, and a subtle radiolucent line in the acromion on plain radiograph was noted (Fig. 3). We prescribed oral analgesics and recommended he stop his exercise regimen. By eight weeks post-surgery, the pain subsided and he complained of protrusion in the area around the posterior acromial border. A new plain radiograph revealed that the radiolucent line at the acromion had widened and the plate hook had cut through the fracture of the acromion (Fig. 4). The patient did not complain of pain, and did not experience any tenderness at the fracture site.

We recommended removal of the plate and osteosynthesis of the acromial fracture with fixation of another plate, as well as an autogenous iliac bone graft because the fracture gap seemed not to be healed. The patient, however, wanted only to have the

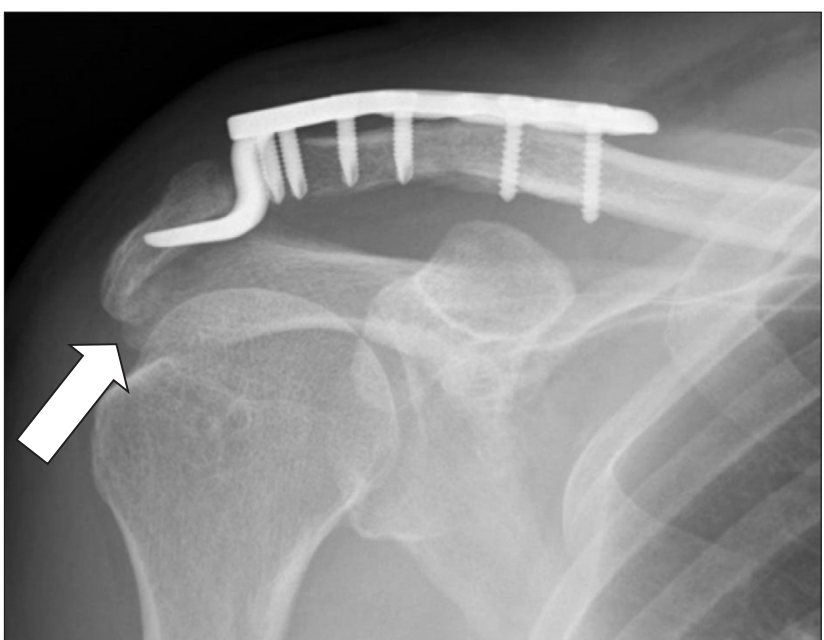

Fig. 3. A radiolucent line in the acromion (white arrow) was noted.

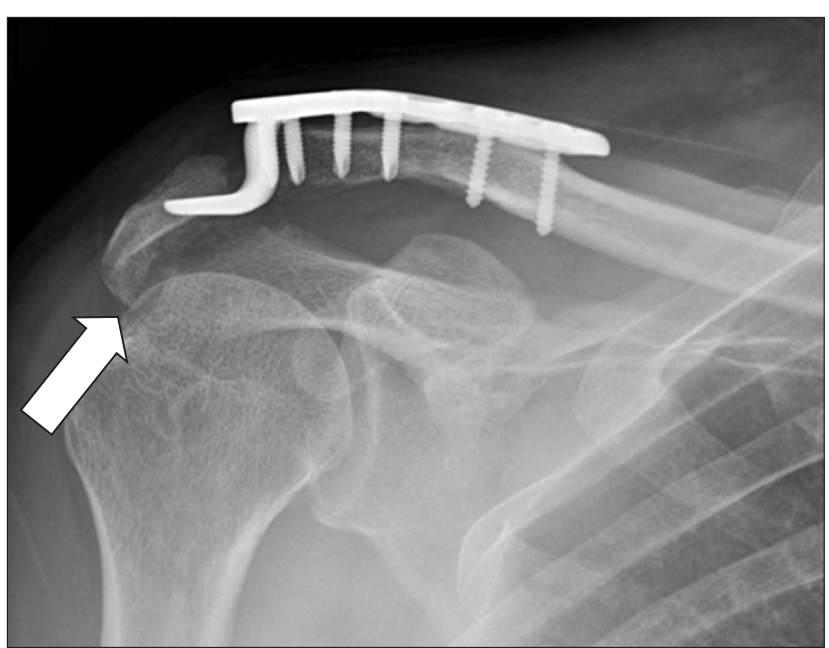

Fig. 4. Widening of the radiolucent line (white arrow) at the acromion was seen and the hook of the plate had cut through the fracture of the acromion.

plate removed because he had no pain. After the removal, the patient underwent computed tomography (CT) imaging to get a more accurate evaluation of the fracture. The fracture had a considerable gap, especially at the anterior and superior fracture sites (Fig. 5). Two months after removal of the hook plate, the patient regained full range of motion of the shoulder without pain. Plain radiographs taken two years after the removal revealed complete bony union of the fracture (Fig. 6) and his right shoulder recovered so completely that the patient could engage in joint-intensive exercises, such as chin-ups, without any pain.

\section{Discussion}

Management of acromioclavicular joint injuries has been controversial and continues to evolve. In cases of Rockwood type $\mathrm{V}$ acromioclavicular dislocations, most physicians have suggested that surgical repair is necessary to avoid complications that may 

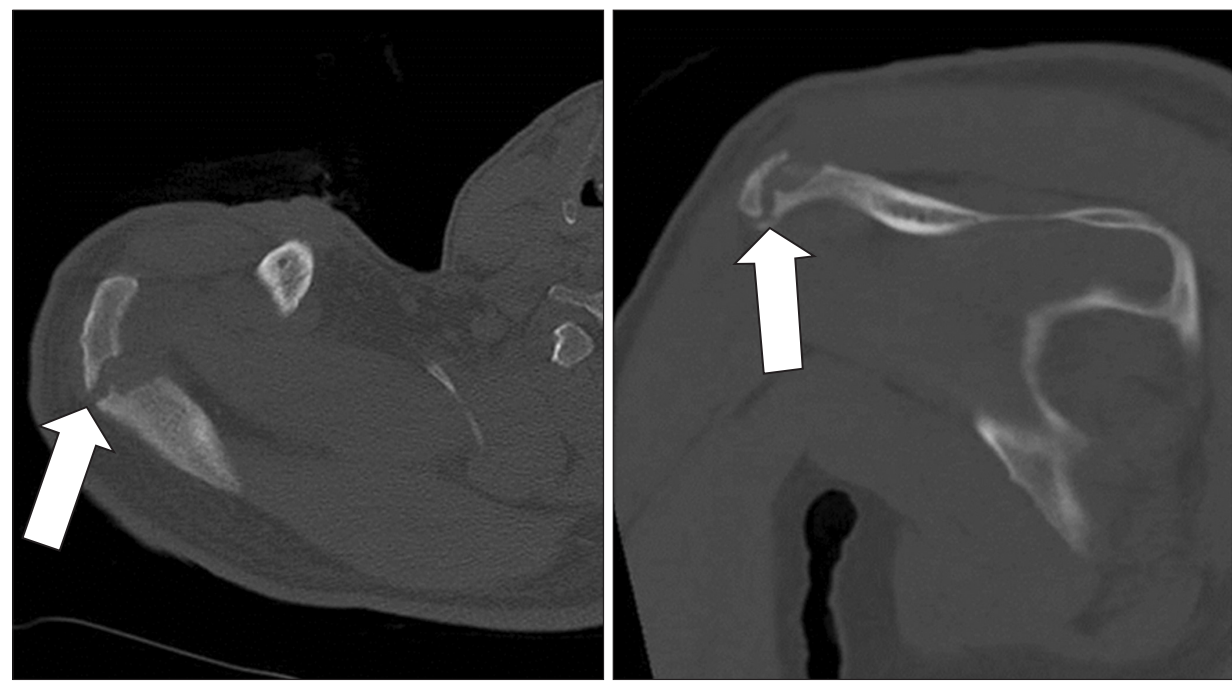

Fig. 5. The anterior and superior fracture gaps (white arrows) were much wider as seen on axial and coronal computed tomography images.

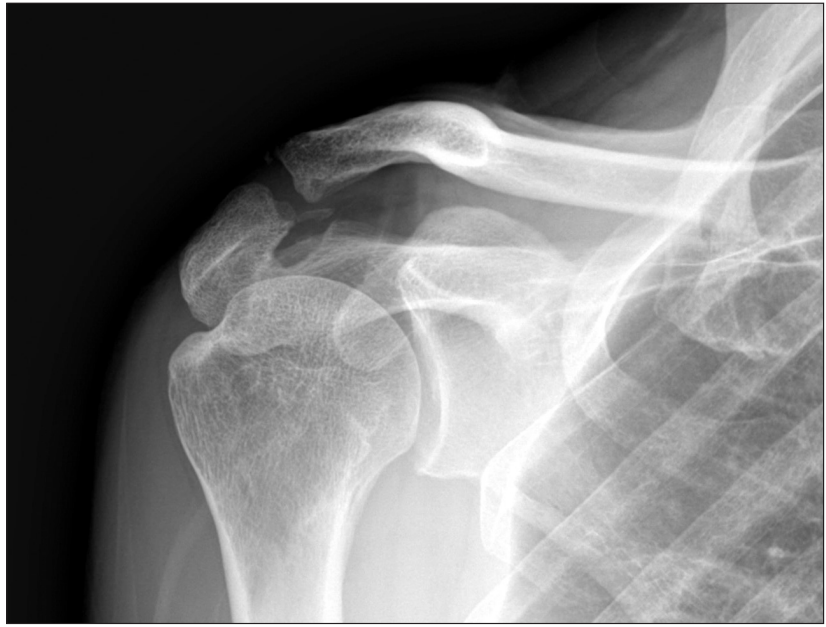

Fig. 6. Complete bony union of the previously fractured acromion was confirmed by plain radiograph.

arise from nonsurgical treatment, such as chronic pain and deformation. ${ }^{4,5)}$ Numerous surgical methods have been described as understanding of shoulder joint biomechanics has increased, and coracoclavicular repair and intra-articular AC fixation are currently the most popular surgical methods for stabilization.

In hook plate fixation, which is a type of intra-articular AC fixation, a lateral hook passes below the acromion, posterior to the AC joint. Positioning of this hook and plate provides distal leverage, bringing the distal clavicle down to facilitate ligament healing and maintain the proper anatomical position of the $\mathrm{AC}$ joint. A small degree of motion between the acromion and the hook of the plate is allowed during shoulder movement, so as not to hinder physiologic movement of the AC joint. The plate should be removed after the ligaments are healed because it can eventually cause complications, such as impingement, osteolysis, and erosion of the acromion. ${ }^{2,6)}$ Preservation of hook motion beneath the acromion, rather than having the plate firmly fixed on the clavicle, is an underlying cause of these complications. ${ }^{7}$

Acromion fracture after clavicular hook plate fixation is a very rare complication, and Chiang et al. ${ }^{3)}$ reported one case of acromion fracture after eight months of hook plate fixation in a case of type V AC dislocation in which the hook plate was not removed. The fate of the acromial fracture was not followed in that case. In this case, we removed the hook plate but the acromial fracture was left without further treatment. Fortunately, bony union of acromial fracture was achieved anyhow; we attribute this to a stable superior shoulder suspensory complex. The coracoclavicular distance did not increase when the acromial fracture was found (Fig. 4), and it was maintained until the fracture was united (Fig. 5). Therefore the superior shoulder suspensory complex was disrupted only at one point (acromial fracture), which could be why a stable superior shoulder suspensory complex was maintained.

The morphometric relationship between the hook and acromion affects stress concentration during the rotational movements of the clavicle. ${ }^{7,8)}$ The hook of the clavicular plate was originally designed to make surface contact with the acromial undersurface, however, it seemed to make point contact between the tip of the hook and the acromial undersurface because of a three-dimensional morphometric mismatch. ${ }^{7}$ Further study is needed to describe the anatomical characteristics of the acromial undersurface and a new design of the hook plate should be developed according to those characteristics.

An acromial stress fracture that was induced by an implanted clavicular hook plate achieved complete bony union without any treatment, despite the presence of a wide gap. This spontaneous healing could be attributed to the patient having a stable superior shoulder suspensory complex. The underlying cause of acromial stress fractures could be that the hook of the plate is a stress riser to the undersurface of the acromion because of morphometric mismatch with the acromial undersurface. 


\section{References}

1. Rolf O, Hann von Weyhern A, Ewers A, Boehm TD, Gohlke F. Acromioclavicular dislocation Rockwood III-V: results of early versus delayed surgical treatment. Arch Orthop Trauma Surg. 2008;128(10):1153-7.

2. Chandrasenan J, Badhe S, Cresswell T, De Beer J. The clavicular hook plate: Consequences in three cases. Eur J Trauma Emerg Surg. 2007;33(5):557-9.

3. Chiang CL, Yang SW, Tsai MY, Kuen-Huang Chen C. Acromion osteolysis and fracture after hook plate fixation for acromioclavicular joint dislocation: a case report. J Shoulder Elbow Surg. 2010;19(4):e13-5.

4. Johansen JA, Grutter PW, McFarland EG, Petersen SA. Acromioclavicular joint injuries: indications for treatment and treatment options. J Shoulder Elbow Surg. 2011;20 2 Suppl:S70-82.

5. Press J, Zuckerman JD, Gallagher M, Cuomo F. Treatment of grade III acromioclavicular separations. Operative versus nonoperative management. Bull Hosp Jt Dis. 1997;56(2):77-83.

6. Haidar SG, Krishnan KM, Deshmukh SC. Hook plate fixation for type II fractures of the lateral end of the clavicle. J Shoulder Elbow Surg. 2006;15(4):419-23.

7. ElMaraghy AW, Devereaux MW, Ravichandiran K, Agur AM. Subacromial morphometric assessment of the clavicle hook plate. Injury. 2010;41(6):613-9.

8. Vähäkari M, Leppilahti J, Hyvönen P, Ristiniemi J, Päivänsalo M, Jalovaara P. Acromial shape in asymptomatic subjects: a study of 305 shoulders in different age groups. Acta Radiol. 2010;51(2):202-6. 